\title{
Families of elliptic curves of high rank with nontrivial torsion group over $\mathbb{Q}$
}

\author{
by \\ LeOpoldo Kulesz (Buenos Aires)
}

Introduction. In 1976, B. Mazur [Maz] proved Beppo Levi's conjecture which asserts that if $E$ is an elliptic curve defined over $\mathbb{Q}$, the only possible torsion groups over $\mathbb{Q}$ are

$$
\begin{cases}\mathbb{Z} / k \mathbb{Z}, & k=2, \ldots, 10 \text { and } 12, \\ \mathbb{Z} / 2 \mathbb{Z} \times \mathbb{Z} / 2 m \mathbb{Z}, & m=1, \ldots, 4\end{cases}
$$

For a finite group $G$ and a number field $\mathbf{K}$, let

$$
\operatorname{Br}(G, \mathbf{K})=\underset{E_{G}}{\limsup } \operatorname{rank}\left(E_{G}(\mathbf{K})\right),
$$

where $E_{G}$ runs through the elliptic curves defined over $\mathbf{K}$ for which $E(\mathbf{K})_{\text {tors }}$ is isomorphic to $G$.

In order to accelerate the factorisation algorithm of H. W. Lenstra [Len], P. L. Montgomery [Mon], H. Suyama [Suy] and A. O. L. Atkin-F. Morain $[\mathrm{A}-\mathrm{M}]$ obtain the following result:

Proposition. $\operatorname{Br}(G, \mathbb{Q}) \geq 1$ for all $G$.

More precisely, for each torsion case they construct an infinite family of elliptic curves over $\mathbb{Q}$ of rank $\geq 1$ parametrised either by the projective line or by another elliptic curve of rank $\geq 1$.

It is natural to ask, for each torsion case, if there exist families of elliptic curves of higher rank.

The case $G=\mathbb{Z} / 2 \mathbb{Z}$ was studied by K. Nagao [Nag] and S. Fermigier $[$ Fer]. Nagao shows that $\operatorname{Br}(\mathbb{Z} / 2 \mathbb{Z}, \mathbb{Q}) \geq 6$ using a family of elliptic curves defined over $\mathbb{Q}$ of rank at least 6 with a rational point of order 2 , parametrised by another elliptic curve of rank $\geq 1$. This result was improved by Fermigier, who showed that $\operatorname{Br}(\mathbb{Z} / 2 \mathbb{Z}, \mathbb{Q}) \geq 8$. He constructed a family of elliptic curves

2000 Mathematics Subject Classification: 11G05, 14H52.

This work was supported by ECOS A99E06. 
defined over $\mathbb{Q}$ of rank at least 8 with a rational of order 2 , parametrised by $\mathbb{Q}\left(t_{1}, \ldots, t_{5}\right)$. He also found in this family a single curve of rank 14 .

Both Nagao and Fermigier obtain their results by applying the method used by J.-F. Mestre in order to find an infinite family of elliptic curves of rank $\geq 12$ [Mes1], [Mes2].

In this paper, we will improve the lower bound of $\operatorname{Br}(G, \mathbb{Q})$ for the other cases of torsion and sharpen the corresponding parametrisations.

\section{PRELIMINARIES}

\subsection{Parametrisation of the elliptic curves with a fixed torsion}

group. In this section we will recall and sometimes reformulate some classic results [Kna], [Kub] and [Na].

Let $E$ be an elliptic curve defined over $\mathbb{Q}$ passing through a $\mathbb{Q}$-rational point $P$. Without loss of generality we can assume $P=(0,0)$; then $E$ admits the following equation on the affine plane:

$$
p(x, y)=y^{2}+a_{1} x y+a_{3} y-\left(x^{3}+a_{2} x^{2}+a_{4} x\right)=0 .
$$

Moreover, since $(\partial p / \partial x)(0,0)=-a_{4}$ and $(\partial p / \partial y)(0,0)=a_{3}, E$ is not singular at $P$ if and only if $a_{3} \neq 0$ or $a_{4} \neq 0$. We will suppose from now on that $E$ is nonsingular at $P$.

The point $P$ is of order 2 if and only if the tangent to $E$ at $P$ is vertical, hence, if and only if $a_{3}=0$, i.e., if and only if $E$ has the equation

$$
y^{2}+a_{1} x y=x^{3}+a_{2} x^{2}+a_{4} x .
$$

Suppose now that $a_{3} \neq 0$. Under the change of coordinates

$$
(x, y) \mapsto\left(X, Y+a_{3}^{-1} a_{4} X\right),
$$

the point $P$ remains invariant and the curve becomes

$$
Y^{2}+\left(a_{1}+2 a_{3}^{-1} a_{4}\right) X Y+a_{3} Y=X^{3}+\left(a_{2}-a_{1} a_{3}^{-1} a_{4}-a_{3}^{-2} a_{4}^{2}\right) X^{2} .
$$

We can rewrite this by changing the notation:

$$
y^{2}+a_{1} x y+a_{3} y=x^{3}+a_{2} x^{2} .
$$

Using the chord-tangent method we obtain

$$
-P=\left(0,-a_{3}\right), \quad[2] P=\left(-a_{2}, a_{1} a_{2}-a_{3}\right) .
$$

As $[3] P=0$ if and only if $-P=[2] P$, we conclude that $P$ is of order 3 if and only if $a_{2}=0$, i.e. $E$ has the equation

$$
y^{2}+a_{1} x y+a_{3} y=x^{3} .
$$

For other cyclic cases of torsion we start directly from Tate's normal form

$$
y^{2}+(1-c) x y-b y=\left(x^{3}-b x^{2}\right),
$$


which can be obtained by the change of coordinates

$$
(x, y) \mapsto\left(X / u^{2}, Y / u^{3}\right) \text { with } u=a_{3}^{-1} a_{2},
$$

and letting $b=-a_{3}^{-2} a_{2}^{3}$ and $c=1-a_{3}^{-1} a_{1} a_{2}$. The chord-tangent method from the point $P=(0,0)$ yields

$$
\begin{gathered}
-P=(0, b), \quad[2] P=(b, b c), \quad[-2] P=(b, 0), \\
{[3] P=(c, b-c), \quad[-3] P=\left(c, c^{2}\right),} \\
{[4] P=\left(\frac{b(b-c)}{c^{2}}, \frac{-b^{2}\left(b-c-c^{2}\right)}{c^{3}}\right), \quad[-4] P=\left(\frac{b(b-c)}{c^{2}}, \frac{(b-c)^{2} b}{c^{3}}\right),} \\
{[5] P=\left(\frac{-b c\left(-c^{2}+b-c\right)}{(b-c)^{2}}, \frac{b c^{2}\left(b^{2}-b c-c^{3}\right)}{(b-c)^{3}}\right),} \\
{[-5] P=\left(\frac{-b c\left(-c^{2}+b-c\right)}{(b-c)^{2}}, \frac{b^{2}\left(-c^{2}+b-c\right)^{2}}{(b-c)^{3}}\right),} \\
{[6] P=\left(\frac{(-b+c)\left(c^{3}+b c-b^{2}\right)}{\left(-b+c+c^{2}\right)^{2}}, \frac{c\left(b c^{2}-c^{2}+3 b c-2 b^{2}\right)(-b+c)^{2}}{\left(-b c+c^{2}\right)^{3}}\right),} \\
{[-6] P=\left(\frac{(-b+c)\left(c^{3}+b c-b^{2}\right)}{\left(-b+c+c^{2}\right)^{2}}, \frac{c\left(c^{3}+b c-b^{2}\right)^{2}}{\left(-b+c+c^{2}\right)^{3}}\right),}
\end{gathered}
$$

and therefore:

(1.1.3) $P$ is of order 4 if and only if $c=0([2] P=[-2] P)$.

(1.1.4) $P$ is of order 5 if and only if $b=c([3] P=[-2] P)$.

(1.1.5) $P$ is of order 6 if and only if $b=c+c^{2}([3] P=[-3] P)$.

(1.1.6) $\quad P$ is of order 7 if and only if $b=d^{3}-d^{2}$ and $c=d^{2}-d$.

(1.1.7) $\quad P$ is of order 8 if and only if

$$
b=(2 d-1)(d-1), \quad c=\frac{(2 d-1)(d-1)}{d} .
$$

(1.1.8) $\quad P$ is of order 9 if and only if

$$
b=c d, \quad c=f d-f, \quad d=f(f-1)+1 .
$$

(1.1.9) $P$ is of order 10 if and only if

$$
b=c d, \quad c=f d-f, \quad d=\frac{f^{2}}{f-(f-1)^{2}} .
$$

(1.1.10) $P$ is of order 12 if and only if

$$
\begin{gathered}
b=c d, \quad c=f d-f, \quad d=m+t, \\
f=\frac{m}{1-t}, \quad m=\frac{3 t-3 t^{2}-1}{t-1} .
\end{gathered}
$$


The cases (1.1.6)-(1.1.10) were obtained from the equalities $[4] P=$ $[-3] P,[4] P=[-4] P,[5] P=[-4] P,[5] P=[-5] P,[5] P=[-6] P$, respectively, which give curves of genus 0 in $b$ and $c$, hence parametrisable.

We suggest a different parametrisation for the cases $\mathbb{Z} / 9 \mathbb{Z}, \mathbb{Z} / 10 \mathbb{Z}$ and $\mathbb{Z} / 12 \mathbb{Z}$, which will be useful later. Instead of considering the conditions that the point $P=(0,0)$ should verify in order to have $[9] P=0$ (resp. $[10] P=0$ and $[12] P=0$ ), we start from the simpler case [3] $P=0$ (resp. [5] $P=0$ and $[6] P=0)$ and look for a point $Q$ such that $[3] Q=P$ (resp. $[2] Q=P$ and $[2] Q=P)$. In this manner we obtain the following results:

- On the elliptic curve defined by the equation

$$
\begin{aligned}
E_{t}: \quad\left(32 t^{2}-8 t\right) y^{2}+\left(-48 t^{2}\right. & \left.+64 t^{3}+1\right) x y \\
& +t(4 t-1) y-8 t x^{3}(4 t-1)=0,
\end{aligned}
$$

the point $Q=\left(t, 2 t^{2} /(4 t-1)\right)$ is of order 9 .

- On the elliptic curve defined by the equation

$$
\begin{aligned}
E_{t}: \quad(t+1)^{2} y^{2} & +\left(2 t^{2}+2 t+1+2 t^{3}\right) x y \\
& +t^{2}(2 t+1) y-(t+1)^{2} x^{3}-t^{2}(2 t+1) x^{2}=0,
\end{aligned}
$$

the point $Q=\left(-t^{2}(2 t+1) /(t+1)^{3},-t^{3}(2 t+1)^{2} /(t+1)^{5}\right)$ is of order 10 .

- On the elliptic curve defined by the equation

$$
\begin{aligned}
E_{t}: \quad y_{1}\left(x_{1}+1\right) y^{2} & +\left(-y_{1}^{2}+2 y_{1}+x_{1}^{3}\right) x y \\
& +\left(-y_{1}^{2}+x_{1}^{3}-2 x_{1} y_{1}\right) y-y_{1}\left(x_{1}+1\right) x^{3}=0
\end{aligned}
$$

with $x_{1}=-(t+1)\left(t^{2}-2 t+5\right) / 8$ and $y_{1}=t\left(1-t^{2}\right) x_{1} / 4$, the point $Q=$ $\left(x_{1}, y_{1}\right)$ is of order 12 .

In order to treat the torsion cases of the form $\mathbb{Z} / 2 \mathbb{Z} \times \mathbb{Z} / 2 n \mathbb{Z}$ we start from the elliptic curve in Weierstrass form:

$$
E: \quad y^{2}=(x-\alpha)(x-\beta)(x-\gamma) .
$$

We know that if $\alpha, \beta$ and $\gamma$ are in $\mathbb{Q}$ then $E(\mathbb{Q})_{\text {tors }}$ contains one torsion subgroup isomorphic to $\mathbb{Z} / 2 \mathbb{Z} \times \mathbb{Z} / 2 \mathbb{Z}$.

In order to study the torsion case $\mathbb{Z} / 2 \mathbb{Z} \times \mathbb{Z} / 4 \mathbb{Z}$ we consider the following result (cf. [Kna, Chapter IV]):

TheOREM 1.1. Let $E$ be an elliptic curve defined over a field $\mathbf{k}$ of characteristic $\neq 2$ or 3 . Suppose that $E$ is given by

$$
y^{2}=(x-\alpha)(x-\beta)(x-\gamma)
$$

with $\alpha, \beta, \gamma \in \mathbf{k}$. For $\left(x_{2}, y_{2}\right)$ in $E(\mathbf{k})$ there exists $\left(x_{1}, y_{1}\right) \in E(\mathbf{k})$ with $[2]\left(x_{1}, y_{1}\right)=\left(x_{2}, y_{2}\right)$ if and only if $x_{2}-\alpha, x_{2}-\beta$ and $x_{2}-\gamma$ are perfect squares in $\mathbf{k}$. 
It follows that the curves $E$ with a torsion subgroup isomorphic to $\mathbb{Z} / 2 \mathbb{Z} \times \mathbb{Z} / 4 \mathbb{Z}$ have the equation

$$
y^{2}=x\left(x+x_{1}^{2}\right)\left(x+x_{2}^{2}\right), \quad x_{1}, x_{2} \in \mathbb{Q} .
$$

Indeed, by applying the theorem, we verify that the point $(0,0)$ is of order 4 .

If we look for $x_{1}$ and $x_{2}$ in the equation (1.1.12) such that the point $\left(x_{1} x_{2}, x_{1} x_{2}\left(x_{1}+x_{2}\right)\right)$ is a double point (cf. Theorem 1.1), we find that the elliptic curves $E$ with a torsion subgroup isomorphic to $\mathbb{Z} / 2 \mathbb{Z} \times \mathbb{Z} / 8 \mathbb{Z}$ have the equation

$$
y^{2}=x\left(x+x_{1}^{2}\right)\left(x+x_{2}^{2}\right)
$$

with $x_{1}=\left(t^{2}-1\right) /(2 t), x_{2}=1 / x_{1}$ and $t \in \mathbb{Q}$. For this last case, it is also possible to start from (1.1.7) and find the parameter $d$ such that this curve has another point of order 2 . It is sufficient to set

$$
d=\frac{-2(4+t)}{-8+t^{2}} \text {. }
$$

Finally, in order to obtain a torsion subgroup isomorphic to $\mathbb{Z} / 2 \mathbb{Z} \times \mathbb{Z} / 6 \mathbb{Z}$, it is sufficient to set $\alpha=x_{1}^{2}, \beta=x_{2}^{2}$ and $\gamma=x_{3}^{2}$ in (1.1.11) and find $x_{1}, x_{2}$ and $x_{3}$ such that the point $\left(0, x_{1} x_{2} x_{3}\right)$ is of order 3 (using (1.1.2)).

Thus, the curves with a torsion subgroup isomorphic to $\mathbb{Z} / 2 \mathbb{Z} \times \mathbb{Z} / 6 \mathbb{Z}$ have the equation

$$
y^{2}=\left(x-x_{1}^{2}\right)\left(x-x_{2}^{2}\right)\left(x-\frac{x_{1}^{2} x_{2}^{2}}{\left(x_{1}-x_{2}\right)^{2}}\right) .
$$

1.2. Transforming a quartic into a cubic. We recall some results about quartics [Cas], [A-M]. Let $E$ be the elliptic curve satisfying the equation

$$
y^{2}=a_{4} x^{4}+a_{3} x^{3}+a_{2} x^{2}+a_{1} x+a_{0}=f(x),
$$

and passing through the rational point $\left(x_{0}, y_{0}\right)$. If we set

$$
x=x_{0}+y_{0}\left(X-\frac{f^{\prime}\left(x_{0}\right)}{4 y_{0}}\right)^{-1}, \quad y=\frac{Y}{y_{0}}\left(x-x_{0}\right)^{2},
$$

we see that $E$ is birationally equivalent to

$$
E^{\prime}: \quad Y^{2}=X^{4}-6 A_{2} X^{2}+4 A_{1} X+A_{0}=F(X) .
$$

This last curve is also birationally equivalent to

$$
E^{\prime \prime}: \quad T^{2}=S^{3}-\frac{3 A_{2}^{2}+A_{0}}{4} S+\frac{A_{1}^{2}-A_{2}\left(A_{2}^{2}-A_{0}\right)}{4},
$$

after the following change of coordinates:

$$
X=\frac{T-A_{1} / 2}{S-A_{2}}, \quad Y=-X^{2}+2 S+A_{2} .
$$


1.3. Independence of a system of points. We consider elliptic curves $E_{x_{1}, \ldots, x_{r}}$ defined over the field $\mathbb{Q}\left(x_{1}, \ldots, x_{r}\right)$; we will have to show that certain points $P_{1}\left(x_{1}, \ldots, x_{r}\right), \ldots, P_{n}\left(x_{1}, \ldots, x_{r}\right)$ are independent on the curve $E_{x_{1}, \ldots, x_{r}}\left(\mathbb{Q}\left(x_{1}, \ldots, x_{r}\right)\right)$. It will be sufficient to find a suitable specialisation $y_{1}, \ldots, y_{r}$ of $x_{1}, \ldots, x_{r}$ in rational values and to show that the points $P_{1}\left(y_{1}, \ldots, y_{r}\right), \ldots, P_{n}\left(y_{1}, \ldots, y_{r}\right)$ are independent on $E_{y_{1}, \ldots, y_{r}}(\mathbb{Q})$ ([Sil $\left.]\right)$. For this, we will compute the matrix of the Néron-Tate heights with gp-PARI [Fer].

\section{RESULTS}

Let us recall some results obtained by Montgomery [Mon], Suyama [Suy] and Atkin-Morain $[\mathrm{A}-\mathrm{M}]$ :

- For $E(\mathbb{Q})_{\text {tors }}$ isomorphic to $\mathbb{Z} / 3 \mathbb{Z}, \mathbb{Z} / 4 \mathbb{Z}, \mathbb{Z} / 5 \mathbb{Z}, \mathbb{Z} / 6 \mathbb{Z}, \mathbb{Z} / 2 \mathbb{Z} \times \mathbb{Z} / 2 \mathbb{Z}$, or $\mathbb{Z} / 2 \mathbb{Z} \times \mathbb{Z} / 4 \mathbb{Z}$, they obtain families of elliptic curves of rank $\geq 1$, parametrised by $\mathbb{Q}(t)$.

- For $E(\mathbb{Q})_{\text {tors }}$ isomorphic to $\mathbb{Z} / 7 \mathbb{Z}, \mathbb{Z} / 8 \mathbb{Z}, \mathbb{Z} / 9 \mathbb{Z}, \mathbb{Z} / 10 \mathbb{Z}, \mathbb{Z} / 12 \mathbb{Z}, \mathbb{Z} / 2 \mathbb{Z} \times$ $\mathbb{Z} / 6 \mathbb{Z}$, or $\mathbb{Z} / 2 \mathbb{Z} \times \mathbb{Z} / 8 \mathbb{Z}$, they obtain families of elliptic curves of rank $\geq 1$, parametrised by an elliptic curve of rank $\geq 1$.

In what follows we improve these results for $E(\mathbb{Q})_{\text {tors }}$ isomorphic to $\mathbb{Z} / 3 \mathbb{Z}, \mathbb{Z} / 4 \mathbb{Z}, \mathbb{Z} / 5 \mathbb{Z}, \mathbb{Z} / 6 \mathbb{Z}, \mathbb{Z} / 7 \mathbb{Z}, \mathbb{Z} / 8 \mathbb{Z}, \mathbb{Z} / 2 \mathbb{Z} \times \mathbb{Z} / 2 \mathbb{Z}, \mathbb{Z} / 2 \mathbb{Z} \times \mathbb{Z} / 4 \mathbb{Z}$ or $\mathbb{Z} / 2 \mathbb{Z} \times \mathbb{Z} / 6 \mathbb{Z}$, either by constructing infinite families of elliptic curves of higher rank or by sharpening the corresponding parametrisation. For $E(\mathbb{Q})_{\text {tors }}$ isomorphic to $\mathbb{Z} / 9 \mathbb{Z}, \mathbb{Z} / 10 \mathbb{Z}, \mathbb{Z} / 12 \mathbb{Z}$ or $\mathbb{Z} / 2 \mathbb{Z} \times \mathbb{Z} / 8 \mathbb{Z}$, we will find parametrisations by other elliptic curves of rank $\geq 1$.

\subsection{The case $E(\mathbb{Q})_{\text {tors }}=\mathbb{Z} / 2 \mathbb{Z} \times \mathbb{Z} / 2 \mathbb{Z}$}

TheOREM 2.1. $\operatorname{Br}(\mathbb{Z} / 2 \mathbb{Z} \times \mathbb{Z} / 2 \mathbb{Z}, \mathbb{Q}) \geq 4$. More precisely, there is an infinite family of elliptic curves of rank at least four, with a torsion subgroup over $\mathbb{Q}$ isomorphic to $\mathbb{Z} / 2 \mathbb{Z} \times \mathbb{Z} / 2 \mathbb{Z}$ and parametrised by $\mathbb{Q}\left(x_{1}, x_{2}, x_{3}, x_{4}\right)$.

Proof. We know that $E$ is an elliptic curve defined over a field $\mathbf{K}$ with a torsion subgroup isomorphic to $\mathbb{Z} / 2 \mathbb{Z} \times \mathbb{Z} / 2 \mathbb{Z}$ if and only if $E$ has a cubic model of the form

$$
y^{2}=(x-\alpha)(x-\beta)(x-\gamma) \quad \text { with } \alpha, \beta, \gamma \in \mathbf{K}
$$

(cf. (1.1.8)). Consider the curves

$$
E_{a, b}: \quad y^{2}=a\left(x^{2}+1\right)^{2}+b x^{2} \quad \text { with } a, b \in \mathbb{Q},
$$

passing through a $\mathbb{Q}$-rational point $\left(x_{0}, y_{0}\right)$. It is easy to verify (cf. 1.2$)$ that these curves have a cubic model of the form

$$
y^{2}=(x-\alpha)(x-\beta)(x-\gamma)
$$


with

$$
\begin{aligned}
& \alpha=-\frac{\left(a x_{0}^{4}+2 a x_{0}^{2}+a-y_{0}^{2}\right)\left(a x_{0}^{4}-2 a x_{0}^{2}-y_{0}^{2}+a\right)}{x_{0}^{2} y_{0}^{4}}, \\
& \beta=-\frac{a\left(x_{0}-1\right)^{2}\left(x_{0}+1\right)^{2}\left(a x_{0}^{4}+2 a x_{0}^{2}+a-y_{0}^{2}\right)}{x_{0}^{2} y_{0}^{4}}, \\
& \gamma=-\frac{a\left(x_{0}^{2}+1\right)^{2}\left(a x_{0}^{4}-2 a x_{0}^{2}-y_{0}^{2}+a\right)}{x_{0}^{2} y_{0}^{4}},
\end{aligned}
$$

and thus, the curves $E_{a, b}$ have a torsion subgroup defined over $\mathbb{Q}$ isomorphic to $\mathbb{Z} / 2 \mathbb{Z} \times \mathbb{Z} / 2 \mathbb{Z}$. In order to obtain such curves we will apply the following method due to J.-F. Mestre [Mes1].

Let $X, X_{1}, X_{2}, X_{3}, X_{4}$ be five indeterminates and $\mathbf{K}=\mathbb{Q}\left(X_{1}, X_{2}, X_{3}, X_{4}\right)$. Let $P \in \mathbf{K}[X]$ be the polynomial $P(X)=\prod_{i=1}^{4}\left(X-X_{i}\right)=X^{4}+c_{3} X^{3}+$ $c_{2} X^{2}+c_{1} X+c_{0}$. It may be written in a unique form as $P=Q^{2}-R$ with $Q$ and $R$ in $\mathbf{K}[X]$ such that $Q(X)=X^{2}+d_{1} X+d_{0}$ and $R(X)=r_{1} X+r_{2}$, where $d_{1}, d_{0}, r_{1}, r_{2} \in \mathbb{Q}$. Indeed, we obtain the equality by setting $d_{1}=c_{3} / 2$, $d_{0}=\left(c_{2}-d_{1}^{2}\right) / 2, r_{1}=2 d_{1} d_{0}-c_{1}$ and $r_{2}=d_{0}^{2}-c_{0}$.

The rational fraction $F_{1}(x)=\left(x^{2}+1\right)^{2} / x^{2}$ is invariant under the action of the group $G_{1}$ of four homographies generated by $x \mapsto-x$ and $x \mapsto 1 / x$. Let $x_{1}, x_{2}, x_{3}$ and $x_{4}$ be four indeterminates. If we set $X_{i}=F_{1}\left(x_{i}\right)$ the numerator of $P\left(F_{1}(x)\right)$ splits completely over $\mathbb{Q}\left(x_{1}, x_{2}, x_{3}, x_{4}\right)$. In this way, we obtain the curve $E_{r_{1}, r_{2}}$ satisfying the equation

$$
y^{2}=r_{1}\left(x^{2}+1\right)^{2}+r_{2} x^{2}
$$

and passing through the points of abscissae $x_{1}, x_{2}, x_{3}$ and $x_{4}$ (and by their conjugates) under the action of $G_{1}$.

When we apply this method to the case where $x_{1}=2, x_{2}=3, x_{3}=4$ and $x_{4}=5$, we obtain the elliptic curve $E$ satisfying the minimal equation

$$
E: \quad y^{2}+x y=x^{3}+a x+b
$$

with

$$
\begin{aligned}
a & =-33266039859280269453163159675, \\
b & =1266432590907122115122625450016203315594257 .
\end{aligned}
$$

It has a torsion subgroup isomorphic to $\mathbb{Z} / 2 \mathbb{Z} \times \mathbb{Z} / 2 \mathbb{Z}$ generated by the points

$$
\begin{aligned}
& P_{1}=(159074830970654,-79537415485327), \\
& P_{2}=(-199067488994146,99533744497073),
\end{aligned}
$$

and passes through the following four independent points (images of the points on $E_{r_{1}, r_{2}}$ of $x$-coordinate $x_{1}=2, x_{2}=3, x_{3}=4$ and $x_{4}=5$ ):

$$
\begin{aligned}
& Q_{1}=(-20566252547452,1393517661684992475371), \\
& Q_{2}=(360529885950854,6011268744207477259073),
\end{aligned}
$$




$$
\begin{aligned}
& Q_{3}=(34589314411754,396442222829819164073), \\
& Q_{4}=(32245757889364,476731254985118349883) .
\end{aligned}
$$

The determinant of the Néron-Tate matrix is 1803.84 (computed with gpPARI), which completes the proof of Theorem 2.1.

\subsection{The case $E(\mathbb{Q})_{\text {tors }}=\mathbb{Z} / 3 \mathbb{Z}$}

TheOREm 2.2. $\operatorname{Br}(\mathbb{Z} / 3 \mathbb{Z}, \mathbb{Q}) \geq 6$. More precisely, there is an infinite family of elliptic curves of rank at least six, with a torsion subgroup over $\mathbb{Q}$ isomorphic to $\mathbb{Z} / 3 \mathbb{Z}$ and parametrised by $\mathbb{Q}\left(x_{1}, x_{2}, x_{3}\right)$.

Proof. By (1.1.2), $E$ is an elliptic curve defined over a field $\mathbf{K}$ with a torsion subgroup over $\mathbf{K}$ isomorphic to $\mathbb{Z} / 3 \mathbb{Z}$ if and only if $E$ has a cubic model of the form

$$
y^{2}+a_{1} x y+a_{3} y=x^{3} \quad \text { with } a_{1}, a_{3} \in \mathbf{K} .
$$

Let $X, X_{1}, X_{2}, X_{3}$ be four indeterminates and $\mathbf{K}=\mathbb{Q}\left(X_{1}, X_{2}, X_{3}\right)$. Let $P(X)=X \prod_{i=1}^{3}\left(X-X_{i}\right)=X^{4}+c_{3} X^{3}+c_{2} X^{2}+c_{1} X \in \mathbf{K}[X]$. Then $P=Q^{2}-R$ for unique $Q$ and $R$ in $\mathbf{K}[X]$ such that $Q(X)=X^{2}+d_{1} X+d_{0}$ and $R(X)=r_{1} X+r_{2}^{2}$, where $d_{1}, d_{0}, r_{1}, r_{2} \in \mathbb{Q}$. Indeed, set $d_{1}=c_{3} / 2$, $d_{0}=\left(c_{2}-d_{1}^{2}\right) / 2, r_{1}=2 d_{1} d_{0}-c_{1}$ and $r_{2}=d_{0}$.

Consider the rational fractions

$$
F_{2}(x)=\frac{x^{3}}{(x+1)^{2}}, \quad g_{2}(x)=-\frac{1}{4} \frac{\left(x^{2}+3\right)^{3}}{(x-1)^{2}(x+1)^{2}},
$$

and three indeterminates $x_{1}, x_{2}$ and $x_{3}$. By setting $X_{i}=g_{2}\left(x_{i}\right)$, the numerator of $P\left(F_{2}(x)\right)$ splits completely over $\mathbb{Q}\left(x_{1}, x_{2}, x_{3}\right)$. In this way, we obtain the curves

$$
E_{r_{1}, r_{2}}: \quad y^{2}=r_{1} x^{3}+r_{2}^{2}(x+1)^{2}
$$

with a torsion subgroup defined over $\mathbb{Q}\left(x_{1}, x_{2}, x_{3}\right)$ isomorphic to $\mathbb{Z} / 3 \mathbb{Z}$. They have a cubic model of the form (cf. 1.2)

$$
E_{r_{1}, r_{2}}^{\prime}: \quad y^{2}-2 r_{2} x y-2 r_{1} r_{2} y=x^{3},
$$

via

$$
E_{r_{1}, r_{2}} \rightarrow E_{r_{1}, r_{2}}^{\prime}, \quad(x, y) \mapsto\left(r_{1} x, r_{1}\left(r_{2}\left(x+r_{1}\right)+y\right)\right) .
$$

Moreover, they pass through the points whose $x$-coordinates are the roots of $F_{2}(x) \prod_{i=1}^{3}\left(F_{2}(x)-g_{2}\left(x_{i}\right)\right)$.

If we apply this method in the case where $x_{1}=2, x_{2}=4$, and $x_{3}=6$, then we obtain the points $P_{1}, \ldots, P_{6}$ of $x$-coordinates $-7,-7 / 9,-19 / 9$, $-19 / 25,-39 / 4,-39 / 49\left(6\right.$ of the 9 roots of $\left.\prod_{i=1}^{3}\left(F_{2}(x)-g_{2}\left(x_{i}\right)\right)\right)$.

We obtain the elliptic curve $E$ of minimal equation

$$
y^{2}+x y=x^{3}+a x+b
$$


with

$$
\begin{aligned}
& a=-78203520427419039841411467, \\
& b=259314050222853661276303764732312995569 .
\end{aligned}
$$

It has a torsion subgroup isomorphic to $\mathbb{Z} / 3 \mathbb{Z}$ generated by the point

$$
P=(7167424811990,8185409686627009297) \text {, }
$$

and passes through the following six independent points (images of the points $\left.P_{1}, \ldots, P_{6}\right)$ :

$$
\begin{aligned}
& Q_{1}=(30967676391166 / 9,150244968139101259355 / 27), \\
& Q_{2}=(-5189102999442,22921483484817715265), \\
& Q_{3}=(7167424811990,-8185416854051821287), \\
& Q_{4}=(52150295496478 / 9,22921402664822970827 / 27), \\
& Q_{5}=(145646473383006 / 25,150244650474432388589 / 125), \\
& Q_{6}=(5762455177454,131221750961285185) .
\end{aligned}
$$

The determinant of the Néron-Tate matrix is 648532.73, which completes the proof of Theorem 2.2.

\subsection{The case $E(\mathbb{Q})_{\text {tors }}=\mathbb{Z} / 4 \mathbb{Z}$}

TheOREM 2.3. $\operatorname{Br}(\mathbb{Z} / 4 \mathbb{Z}, \mathbb{Q}) \geq 3$. More precisely, there is an infinite family of elliptic curves of rank at least three, with a torsion subgroup over $\mathbb{Q}$ isomorphic to $\mathbb{Z} / 4 \mathbb{Z}$ and parametrised by $\mathbb{Q}\left(x_{1}, x_{2}, x_{3}\right)$.

Proof. By (1.1.3), $E$ is an elliptic curve defined over a field $\mathbf{K}$ with a torsion subgroup over $\mathbf{K}$ isomorphic to $\mathbb{Z} / 4 \mathbb{Z}$ if and only if $E$ has a cubic model of the form

$$
y^{2}+x y-b y=x^{3}-b x^{2} \quad \text { with } b \in \mathbf{K} .
$$

We proceed as in Theorem 2.2, this time with the rational fraction $F_{3}(x)=$ $x^{2} /(x-1)$. If we set $X_{i}=F_{3}\left(x_{i}\right)$, the numerator of $P\left(F_{3}(x)\right)$ splits completely over $\mathbb{Q}\left(x_{1}, x_{2}, x_{3}\right)$. In this way, we obtain the curves

$$
E_{r_{1}, r_{2}}: \quad y^{2}=r_{1} x^{2}(x-1)+r_{2}^{2}(x-1)^{2}
$$

with a torsion subgroup defined over $\mathbb{Q}\left(x_{1}, x_{2}, x_{3}\right)$ isomorphic to $\mathbb{Z} / 4 \mathbb{Z}$. Indeed, they have a cubic model of the form (cf. 1.2)

$$
E_{r_{1}, r_{2}}^{\prime}: \quad y^{2}-2(x-b) y=x^{3}-b x^{2} \quad \text { with } b=r_{1} / r_{2}^{2},
$$

via

$$
E_{r_{1}, r_{2}} \rightarrow E_{r_{1}, r_{2}}^{\prime}, \quad(x, y) \mapsto\left(b x, b\left(x-1+y / r_{2}\right)\right) .
$$

Moreover, they pass through the points whose $x$-coordinates are the roots of $F_{2}(x) \prod_{i=1}^{3}\left(F_{2}(x)-g_{2}\left(x_{i}\right)\right)$. 
Applying this method to the case where $x_{1}=3, x_{2}=4$, and $x_{3}=5$, we obtain the elliptic curve $E$ of minimal equation

$$
y^{2}+x y=x^{3}+a x+b
$$

with

$$
a=-266721356141, \quad b=52307554376730321 .
$$

It has a torsion group isomorphic to $\mathbb{Z} / 4 \mathbb{Z}$ generated by the point

$$
P=(554026,272839207) \text {, }
$$

and passes through the following three independent points (images of the points on $E_{r_{1}, r_{2}}$ of $x$-coordinates $x_{1}, x_{2}$ and $\left.x_{3}\right)$ :

$$
\begin{aligned}
& Q_{1}=(249930,35340231), \\
& Q_{2}=(268936,5139697), \\
& Q_{3}=(211918,72706027) .
\end{aligned}
$$

The determinant of the Néron-Tate matrix is 43.88 , which completes the proof of Theorem 2.3 .

\subsection{The case $E(\mathbb{Q})_{\text {tors }}=\mathbb{Z} / 5 \mathbb{Z}$}

TheOREm 2.4. $\operatorname{Br}(\mathbb{Z} / 5 \mathbb{Z}, \mathbb{Q}) \geq 2$. More precisely, there is an infinite family of elliptic curves of rank at least two, with a torsion subgroup over $\mathbb{Q}$ isomorphic to $\mathbb{Z} / 5 \mathbb{Z}$ and parametrised by $\mathbb{Q}(t)$.

Proof. By (1.1.4), $E$ is an elliptic curve defined over a field $\mathbf{K}$ with a torsion subgroup over $\mathbf{K}$ isomorphic to $\mathbb{Z} / 5 \mathbb{Z}$ if and only if $E$ has a cubic model of the form

$$
E_{b}: \quad y^{2}+(1-b) x y-b y=x^{3}-b x^{2} \quad \text { with } b \in \mathbf{K} .
$$

Set

$$
\begin{aligned}
& b=\frac{-\left(3 t^{2}+6 t+4\right)\left(t^{2}+6 t+12\right)}{(t-2)^{2}(t+2)^{2}}, \\
& u=\frac{-\left(8+8 t+t^{2}\right)}{(t-2)(t+2)} \\
& v=\frac{-\left(t^{2}+6 t+12\right)}{(t-2)(t+2)}
\end{aligned}
$$

We will show that the points $P_{1}=(-1, u)$ and $P_{2}=(v, v)$ are independent in $E_{b}(\mathbb{Q}(t))$. If $t=4$, we obtain the elliptic curve $E$ of minimal equation

$$
y^{2}+y=x^{3}+x^{2}+a x+b
$$

with

$$
a=-112845920, \quad b=461373286640 .
$$


It has a torsion subgroup isomorphic to $\mathbb{Z} / 5 \mathbb{Z}$ generated by the point

$$
P=(6202,10003),
$$

and passes through the following two independent points (images of $P_{1}$ and $\left.P_{2}\right)$ :

$$
Q_{1}=(6121,3766), \quad Q_{2}=(5851,38083) .
$$

The determinant of the Néron-Tate matrix is 11.74, which completes the proof of Theorem 2.4.

\subsection{The case $E(\mathbb{Q})_{\text {tors }}=\mathbb{Z} / 6 \mathbb{Z}$}

Theorem 2.5. $\operatorname{Br}(\mathbb{Z} / 6 \mathbb{Z}, \mathbb{Q}) \geq 2$. More precisely, there is an infinite family of elliptic curves of rank at least two, with a torsion subgroup over $\mathbb{Q}$ isomorphic to $\mathbb{Z} / 6 \mathbb{Z}$ and parametrised by $\mathbb{Q}(t)$.

Proof. By (1.1.5), $E$ is an elliptic curve defined over a field $\mathbf{K}$ with a torsion subgroup over $\mathbf{K}$ isomorphic to $\mathbb{Z} / 6 \mathbb{Z}$ if and only if $E$ has a cubic model of the form

$$
E_{c}: \quad y^{2}+(1-c) x y-\left(c+c^{2}\right) y=x^{3}-\left(c+c^{2}\right) x^{2} \quad \text { with } c \in \mathbf{K} .
$$

Set

$$
c=\frac{4(t-1)\left(-2 t+1+2 t^{2}\right)}{5-8 t+4 t^{4}} .
$$

We will show that the points $P_{1}$ and $P_{2}$ of $x$-coordinate $-c$ and $c t$ respectively are independent in $E_{c}(\mathbb{Q}(t))$. If $t=2$, we obtain the elliptic curve $E$ of minimal equation

$$
y^{2}+x y=x^{3}+a x+b
$$

with

$$
a=-1747020, \quad b=867156112 .
$$

It has a torsion subgroup isomorphic to $\mathbb{Z} / 6 \mathbb{Z}$ generated by the point

$$
P=(-396,38888)
$$

and passes through the following two independent points (images of $P_{1}$ and $P_{2}$ ):

$$
Q_{1}=(-1456,18748), \quad Q_{2}=(1724,53728) .
$$

The determinant of the Néron-Tate matrix is 6.47 , which completes the proof of Theorem 2.5.

\subsection{The case $E(\mathbb{Q})_{\text {tors }}=\mathbb{Z} / 7 \mathbb{Z}$}

TheOREM 2.6. $\operatorname{Br}(\mathbb{Z} / 7 \mathbb{Z}, \mathbb{Q}) \geq 1$. More precisely, there is an infinite family of elliptic curves of rank at least one, with a torsion subgroup over $\mathbb{Q}$ isomorphic to $\mathbb{Z} / 7 \mathbb{Z}$ and parametrised by $\mathbb{Q}(t)$. 
Proof. By (1.1.6), $E$ is an elliptic curve defined over a field $\mathbf{K}$ with a torsion subgroup over $\mathbf{K}$ isomorphic to $\mathbb{Z} / 7 \mathbb{Z}$, if and only if $E$ has a cubic model of the form

$$
E_{d}: \quad y^{2}+(1-c) x y-b y=x^{3}-b x^{2}
$$

with $b=d^{3}-d^{2}, c=d^{2}-d$ and $d \in \mathbf{K}$. Set

$$
d=\frac{-2(-3+t)}{3+t^{2}}
$$

The point of abscissa

$$
\frac{-2(t-1)(t+3)(t+1)(-3+t)^{2}}{\left(3+t^{2}\right)^{3}}
$$

is of infinite order in $E_{d}(\mathbb{Q}(t))$ since it is not in $E_{d}(\mathbb{Q}(t))_{\text {tors }}$, except for a finite set of rational values of $t$, which completes the proof of Theorem 2.6.

\subsection{The case $E(\mathbb{Q})_{\text {tors }}=\mathbb{Z} / 8 \mathbb{Z}$}

TheOREM 2.7. $\operatorname{Br}(\mathbb{Z} / 8 \mathbb{Z}, \mathbb{Q}) \geq 1$. More precisely, there is an infinite family of elliptic curves of rank at least one, with a torsion subgroup over $\mathbb{Q}$ isomorphic to $\mathbb{Z} / 8 \mathbb{Z}$ and parametrised by $\mathbb{Q}(t)$.

Proof. By (1.1.7), $E$ is an elliptic curve defined over a field $\mathbf{K}$ with a torsion subgroup over $\mathbf{K}$ isomorphic to $\mathbb{Z} / 8 \mathbb{Z}$ if and only if $E$ has a cubic model of the form

$$
E_{d}: \quad y^{2}+(1-c) x y-b y=x^{3}-b x^{2}
$$

with $b=(2 d-1)(d-1), c=(2 d-1)(d-1) / d$ and $d \in \mathbf{K}$. Set $d=$ $\left(2-2 t+t^{2}\right) /\left(2+t^{2}\right)$.

The point of abscissa

$$
\frac{-2 t\left(2-4 t+t^{2}\right)\left(t^{2}-2\right)}{\left(2+t^{2}\right)^{2}\left(2-2 t+t^{2}\right)}
$$

is of infinite order in $E_{d}(\mathbb{Q}(t))$ since it is not in $E_{d}(\mathbb{Q}(t))_{\text {tors }}$, and $E_{d}(\mathbb{Q}(t))_{\text {tors }}$ is isomorphic to $\mathbb{Z} / 2 \mathbb{Z} \times \mathbb{Z} / 8 \mathbb{Z}$ only for a finite number of values of $t$, which completes the proof of Theorem 2.7 .

\subsection{The case $E(\mathbb{Q})_{\text {tors }}=\mathbb{Z} / 2 \mathbb{Z} \times \mathbb{Z} / 4 \mathbb{Z}$}

TheOREM 2.8. $\operatorname{Br}(\mathbb{Z} / 2 \mathbb{Z} \times \mathbb{Z} / 4 \mathbb{Z}, \mathbb{Q}) \geq 2$. More precisely, there is an infinite family of elliptic curves of rank at least two, with a torsion subgroup over $\mathbb{Q}$ isomorphic to $\mathbb{Z} / 2 \mathbb{Z} \times \mathbb{Z} / 4 \mathbb{Z}$ and parametrised by $\mathbb{Q}\left(t_{1}, t_{2}, t_{3}\right)$.

Proof. By (1.1.9), $E$ is an elliptic curve defined over a field $\mathbf{K}$ with a torsion subgroup over $\mathbf{K}$ isomorphic to $\mathbb{Z} / 2 \mathbb{Z} \times \mathbb{Z} / 4 \mathbb{Z}$ if and only if $E$ has a 
cubic model of the form

$$
E_{u_{1}, u_{2}}: \quad y^{2}=x\left(x+u_{1}^{2}\right)\left(x+u_{2}^{2}\right) \quad \text { with } u_{1}, u_{2} \in \mathbf{K} .
$$

Let $x_{1}, x_{2} \in \mathbb{Q}$. How could we find $u_{1}, u_{2}, y_{1}$ and $y_{2}$ in $\mathbb{Q}$ such that $\left(x_{i}^{2}+u_{1}^{2}\right)\left(x_{i}^{2}+u_{2}^{2}\right)=y_{i}^{2}(i=1,2)$ ?

If we consider $E_{u_{1}, u_{2}}$ as a conic in $y$ and $u_{2}$, it is easy to see that we can answer this question by setting

$$
\begin{aligned}
u_{2} & =\frac{s^{2} u_{1}-2 s u_{1}^{2}-2 x_{1}^{2} s+u_{1} x_{1}^{2}+u_{1}^{3}}{s^{2}-x_{1}^{2}-u_{1}^{2}}, \\
s & =\frac{1}{2} \frac{x_{2}^{2} x_{1}^{2}+u_{1}^{4}+2 x_{2}^{2} u_{1}^{2}}{u_{1}\left(x_{2}^{2}+u_{1}^{2}\right)} .
\end{aligned}
$$

In this manner, we construct an infinite family of elliptic curves

$$
E_{u_{1}, u_{2}}: \quad y^{2}=x\left(x+u_{1}^{2}\right)\left(x+u_{2}^{2}\right)
$$

with $u_{2} \in \mathbb{Q}\left(x_{1}, x_{2}, u_{1}\right)$, and passing through the points with the $x$-coordinate given by $x_{1}^{2}$ and $x_{2}^{2}$.

The points $P_{1}$ and $P_{2}$ with $x$-coordinates 4 and $t^{2}$ are independent in $E_{t}(\mathbb{Q}(t))$. If $t=5$, we obtain the elliptic curve $E$ satisfying the minimal equation

$$
y^{2}=x^{3}+a x^{2}+b x
$$

with

$$
a=1866892562, \quad b=153388875753868561 .
$$

It has a torsion subgroup isomorphic to $\mathbb{Z} / 2 \mathbb{Z} \times \mathbb{Z} / 4 \mathbb{Z}$ generated by the points

$$
R_{1}=(-86136961,0), \quad R_{2}=(391648919,20162086350120)
$$

and passes through the following two independent points (images of $P_{1}$ and $P_{2}$ ):

$$
\begin{aligned}
& Q_{1}=(344547844,17758857249370), \\
& Q_{2}=(2153424025,137744198443930) .
\end{aligned}
$$

The determinant of the Néron-Tate matrix is 112.65 , which completes the proof of Theorem 2.8 .

\subsection{The case $E(\mathbb{Q})_{\text {tors }}=\mathbb{Z} / 2 \mathbb{Z} \times \mathbb{Z} / 6 \mathbb{Z}$}

TheORem 2.9. $\operatorname{Br}(\mathbb{Z} / 2 \mathbb{Z} \times \mathbb{Z} / 6 \mathbb{Z}, \mathbb{Q}) \geq 1$. More precisely, there is an infinite family of elliptic curves of rank at least one, with a torsion subgroup over $\mathbb{Q}$ isomorphic to $\mathbb{Z} / 2 \mathbb{Z} \times \mathbb{Z} / 6 \mathbb{Z}$ and parametrised by $\mathbb{Q}(t)$.

Proof. By (1.1.10), $E$ is an elliptic curve defined over a field $\mathbf{K}$ with a torsion subgroup over $\mathbf{K}$ isomorphic to $\mathbb{Z} / 2 \mathbb{Z} \times \mathbb{Z} / 6 \mathbb{Z}$ if and only if $E$ has a 
cubic model of the form

$$
E_{x_{1}, x_{2}}: \quad y^{2}=\left(x+x_{1}^{2}\right)\left(x+x_{2}^{2}\right)\left(x+\frac{x_{1}^{2} x_{2}^{2}}{\left(x_{1}-x_{2}\right)^{2}}\right) \quad \text { with } x_{1}, x_{2} \in \mathbf{K} .
$$

Set

$$
x_{1}=-\frac{1+2 t}{(t-1)(t+1)}, \quad x_{2}=x_{1}^{2} .
$$

The point whose $x$-coordinate is $x_{1}^{3}$ is of infinite order in $E_{x_{1}, x_{2}}(\mathbb{Q}(t))$ since it is not in $E_{x_{1}, x_{2}}(\mathbb{Q}(t))_{\text {tors }}$, except for a finite number of rational values of $t$, which completes the proof of Theorem 2.9.

2.10. The case $E(\mathbb{Q})_{\text {tors }}=\mathbb{Z} / 9 \mathbb{Z}$. In the first section we found two different parametrisations of elliptic curves defined over a field $\mathbf{K}$ with a torsion subgroup over $\mathbf{K}$ isomorphic to $\mathbb{Z} / 9 \mathbb{Z}$ :

$$
E_{f}: \quad y^{2}+(1-c) x y-b y=\left(x^{3}-b x^{2}\right)
$$

with $b=c d, c=f d-f$ and $d=f(f-1)+1$ (cf. (1.1.8)) and

$E_{t}: \quad\left(32 t^{2}-8 t\right) y^{2}+\left(-48 t^{2}+64 t^{3}+1\right) x y+t(4 t-1) y-8 t x^{3}(4 t-1)=0$

(cf. $\left.\left(1.1 .8^{\prime}\right)\right)$. We consider the following elliptic curves:

$$
\begin{array}{ll}
E_{1}: & y^{2}=(x-2)\left(x^{3}-4 x^{2}+x-2\right) \\
E_{2}: & y^{2}=x(4 x+1)\left(4 x^{2}-7 x+1\right) \\
E_{3}: & y^{2}=-(2 x-1)\left(32 x^{2}-2 x-1\right) \\
E_{4}: & y^{2}=-(8 x-1)(4 x-1)\left(32 x^{2}-20 x-1\right) .
\end{array}
$$

The point $(0,2)$ (resp. $(-1 / 4,0),(1 / 4,1 / 2),(1 / 8,0))$ is of infinite order in $E_{1}(\mathbb{Q})\left(\operatorname{resp.} E_{2}(\mathbb{Q}), E_{3}(\mathbb{Q}), E_{4}(\mathbb{Q})\right)$ and hence $E_{1}\left(\right.$ resp. $\left.E_{2}, E_{3}, E_{4}\right)$ has rank $\geq 1$ over $\mathbb{Q}$.

Theorem 2.10. $E_{1}(\mathbb{Q}), E_{2}(\mathbb{Q}), E_{3}(\mathbb{Q})$ and $E_{4}(\mathbb{Q})$ parametrise elliptic curves with a torsion subgroup over $\mathbb{Q}$ isomorphic to $\mathbb{Z} / 9 \mathbb{Z}$, of rank $\geq 1$.

Proof. On $E_{f},[6](0,0)=(u(f), v(f))$ with

$$
u(f)=f^{2}(f-1), \quad v(f)=f^{4}(f-1)^{2} .
$$

Hence, if we set

$$
p(x, y)=y^{2}+(1-c) x y-b y-\left(x^{3}-b x^{2}\right)
$$

with $b=c d, c=f d-f$ and $d=f(f-1)+1$, then the polynomial $p(x, v(f))$ vanishes at $x=u(f)$. In this way, $p(x, v(f)) /(x-u(f))$ is a polynomial of degree 2 in $x$ and splits in $\mathbb{Q}$ if and only if $(f-2)\left(f^{3}-f x^{2}+f-2\right)$ is a square in $\mathbb{Q}$, i.e. if and only if $f$ is the abscissa of a point of $E_{1}(\mathbb{Q})$. The roots of this polynomial are the $x$-coordinates of points of infinite order of $E_{f}(\mathbb{Q})$. 
For $E_{2}, E_{3}$ and $E_{4}$ we apply the same idea to $E_{t}$ with $P=\left(t, 2 t^{2} /(4 t-1)\right)$,

$$
[4] P=\left(\frac{-1}{4(4 t-1)}, \frac{-1}{32 t(4 t-1)}\right) \quad \text { and } \quad[5] P=\left(\frac{-1}{4(4 t-1)}, \frac{-1}{2 t(4 t-1)^{2}}\right)
$$

respectively.

2.11. The case $E(\mathbb{Q})_{\text {tors }}=\mathbb{Z} / 10 \mathbb{Z}$. In the first section we found two different parametrisations of elliptic curves defined over a field $\mathbf{K}$ with a torsion subgroup over $\mathbf{K}$ isomorphic to $\mathbb{Z} / 10 \mathbb{Z}$ :

$$
E_{f}: \quad y^{2}+(1-c) x y-b y=\left(x^{3}-b x^{2}\right)
$$

with $b=c d, c=f d-f$ and $d=f^{2} /\left(f-(f-1)^{2}\right)($ cf. (1.1.9)), and

$$
\begin{aligned}
E_{t}: \quad(t+1)^{2} y^{2}+\left(2 t^{2}+2 t+1+2 t^{3}\right) & x y+t^{2}(2 t+1) y \\
& -(t+1)^{2} x^{3}-t^{2}(2 t+1) x^{2}=0
\end{aligned}
$$

(cf. $\left.\left(1.1 .9^{\prime}\right)\right)$. We consider the following elliptic curves:

$$
\begin{array}{ll}
E_{1}: & y^{2}=(x-2)(x+1)\left(x^{2}-5 x+2\right), \\
E_{2}: & y^{2}=2 x^{3}+2 x^{2}+2 x+1, \\
E_{3}: & y^{2}=\left(1-3 x-4 x^{2}+4 x^{3}\right)(x+1), \\
E_{4}: & y^{2}=5 x^{4}+8 x^{3}+12 x^{2}+12 x+4 .
\end{array}
$$

The point $(-1,0)$ (resp. $(0,1),(-1,0),(-1,1))$ is of infinite order in $E_{1}(\mathbb{Q})$ $\left(\right.$ resp. $\left.E_{2}(\mathbb{Q}), E_{3}(\mathbb{Q}), E_{4}(\mathbb{Q})\right)$ and thus $E_{1}$ (resp. $\left.E_{2}, E_{3}, E_{4}\right)$ has rank $\geq 1$ over $\mathbb{Q}$.

Theorem 2.11. $E_{1}(\mathbb{Q}), E_{2}(\mathbb{Q}), E_{3}(\mathbb{Q})$ and $E_{4}(\mathbb{Q})$ parametrise elliptic curves with a torsion subgroup over $\mathbb{Q}$ isomorphic to $\mathbb{Z} / 10 \mathbb{Z}$, of rank $\geq 1$.

Proof. On $E_{f},[6](0,0)=(u(f), v(f))$ with

$$
u(f)=\frac{f^{2}(2 f-1)(f-1)}{\left(-3 f+f^{2}+1\right)^{2}}, \quad v(f)=\frac{-f^{2}(2 f-1)^{2}(f-1)^{2}}{\left(-3 f+f^{2}+1\right)^{3}} .
$$

Hence, if we set

$$
p(x, y)=y^{2}+(1-c) x y-b y-\left(x^{3}-b x^{2}\right)
$$

with $b=c d, c=f d-f$ and $d=f^{2} /\left(f-(f-1)^{2}\right)$, then the polynomial $p(x, v(f))$ vanishes at $x=u(f)$. In this way, $p(x, v(f)) /(x-u(f))$ is a polynomial of degree 2 in $x$ and splits in $\mathbb{Q}$ if and only if $(f-2)(f+1)\left(f^{2}-5 f+2\right)$ is a square in $\mathbb{Q}$, i.e. if and only if $f$ is the $x$-coordinate of a point of $E_{1}(\mathbb{Q})$. The roots of this polynomial are the $x$-coordinates of points of infinite order of $E_{f}(\mathbb{Q})$.

For $E_{2}, E_{3}$ and $E_{4}$ we apply the same idea to $E_{t}$ with

$$
[2] P=\left(\frac{-t^{2}(2 t+1)}{(t+1)^{2}}, \frac{t^{4}(2 t+1)^{2}}{(t+1)^{4}}\right),
$$




$$
\begin{aligned}
& {[3] P=\left(\frac{t(2 t+1)}{t+1}, \frac{t^{2}(2 t+1)^{2}}{(t+1)^{3}}\right),} \\
& {[5] P=\left(-t^{2}, \frac{t^{4}}{t+1}\right)}
\end{aligned}
$$

respectively, where

$$
P=\left(\frac{-t^{2}(2 t+1)}{(t+1)^{3}}, \frac{-t^{3}(2 t+1)^{2}}{(t+1)^{5}}\right)
$$

2.12. The case $E(\mathbb{Q})_{\text {tors }}=\mathbb{Z} / 12 \mathbb{Z}$. In the first section we parametrised the elliptic curves defined over a field $\mathbf{K}$ with a torsion subgroup over $\mathbf{K}$ isomorphic to $\mathbb{Z} / 12 \mathbb{Z}$, in the following way (cf. $\left(1.1 .10^{\prime}\right)$ ):

$$
\begin{aligned}
E_{t}: \quad x_{1} y_{1}\left(x_{1}+1\right) & y^{2}+\left(-y_{1}^{2} x_{1}-2 x_{1} y_{1}+x_{1}^{2} x_{1}^{2}\right) x y \\
& +x_{1}\left(-y_{1}^{2}+x_{1} x_{1}^{2}+2 x_{1} y_{1}\right) y-x_{1} y_{1}\left(x_{1}+1\right) x^{3}=0
\end{aligned}
$$

with $x_{1}=-(t+1)\left(t^{2}-2 t+5\right) / 8$ and $y_{1}=t\left(1-t^{2}\right) / 4$.

We consider the following elliptic curve:

$$
E_{1}: \quad y^{2}=\left(x^{4}+6 x^{3}-24 x^{2}+90 x-9\right) .
$$

The point $(1,8)$ is of infinite order in $E_{1}(\mathbb{Q})$ and thus $E_{1}$ has rank $\geq 1$ over $\mathbb{Q}$.

THEOREM 2.12. $E_{1}(\mathbb{Q})$ parametrises elliptic curves with a torsion subgroup over $\mathbb{Q}$ isomorphic to $\mathbb{Z} / 12 \mathbb{Z}$, of rank $\geq 1$.

Proof. On $E_{t},[9]\left(x_{1}, y_{1}\right)=(u(t), v(t))$ with

$$
u(t)=\frac{\frac{1}{4}\left(t^{2}-2 t+5\right)(t+1)^{2}}{(t-1)^{2}}, \quad v(t)=\frac{\frac{1}{16}\left(t^{2}-2 t+5\right)^{2}(t+1)^{4}}{(t-1)^{4}} .
$$

Thus, if we set

$$
\begin{aligned}
p(x, y)= & x_{1} y_{1}\left(x_{1}+1\right) y^{2}+\left(-y_{1}^{2} x_{1}-2 x_{1} y_{1}+x_{1}^{2} x_{1}^{2}\right) x y \\
& +x_{1}\left(-y_{1}^{2}+x_{1} x_{1}^{2}+2 x_{1} y_{1}\right) y-x_{1} y_{1}\left(x_{1}+1\right) x^{3},
\end{aligned}
$$

with

$$
b=(2 d-1)(d-1), \quad c=\frac{(2 d-1)(d-1)}{d}, \quad d=\frac{-2(4+t)}{-8+t^{2}},
$$

the polynomial $p(x, v(t))$ vanishes at $x=u(t)$. Hence, $p(x, v(t)) /(x-v(t))$ is a polynomial of degree 2 in $x$ and splits in $\mathbb{Q}$ if and only if $t^{4}+6 t^{3}-$ $24 t^{2}+90 t-9$ is a square in $\mathbb{Q}$, i.e. if and only if $t$ is the $x$-coordinate of a point of $E_{1}(\mathbb{Q})$. The roots of this polynomial are the $x$-coordinates of points of infinite order of $E_{t}(\mathbb{Q})$. 
2.13. The case $E(\mathbb{Q})_{\text {tors }}=\mathbb{Z} / 2 \mathbb{Z} \times \mathbb{Z} / 8 \mathbb{Z}$. In the first section we parametrised the elliptic curves defined over a field $\mathbf{K}$ with a torsion subgroup over $\mathbf{K}$ isomorphic to $\mathbb{Z} / 12 \mathbb{Z}$ in the following way (cf. $\left(1.1 .13^{\prime}\right)$ ):

$$
E_{t}: \quad y^{2}+(1-c) x y-b y=x^{3}-b x^{2}
$$

with

$$
b=(2 d-1)(d-1), \quad c=\frac{(2 d-1)(d-1)}{d}, \quad d=\frac{-2(4+t)}{-8+t^{2}} .
$$

Define the elliptic curve

$$
E_{1}: \quad y^{2}=-\left(x^{4}+8 x^{3}+24 x^{2}-64\right) .
$$

The point $(-2,4)$ is of infinite order in the curve $E_{1}(\mathbb{Q})$ and hence $E_{1}$ has rank $\geq 1$ over $\mathbb{Q}$.

THEOREM 2.13. $E_{t}(\mathbb{Q})$ parametrises elliptic curves with a torsion subgroup over $\mathbb{Q}$ isomorphic to $\mathbb{Z} / 2 \mathbb{Z} \times \mathbb{Z} / 8 \mathbb{Z}$, of rank $\geq 1$.

Proof. On $E_{t},[3](0,0)=(u(t), v(t))$ with

$$
u(t)=\frac{\left(8+4 t+t^{2}\right) t(2+t)}{\left(-8+t^{2}\right)^{2}}, \quad v(t)=\frac{-\frac{1}{2} t^{2}(2+t)^{2}\left(8+4 t+t^{2}\right)^{2}}{(4+t)\left(-8+t^{2}\right)^{3}} .
$$

Thus, if we let

$$
p(x, y)=y^{2}+(1-c) x y-b y-\left(x^{3}-b x^{2}\right)
$$

with

$$
b=(2 d-1)(d-1), \quad c=\frac{(2 d-1)(d-1)}{d}, \quad d=\frac{-2(4+t)}{-8+t^{2}},
$$

the polynomial $p(x, v(t))$ vanishes at $x=u(t)$. It follows that the polynomial $p(x, v(t)) /(x-u(t))$ is of degree 2 in $x$ and it splits in $\mathbb{Q}$ if and only if $-\left(t^{4}+8 t^{3}+24 t^{2}-64\right)$ is a square in $\mathbb{Q}$, i.e. if and only if $t$ is the $x$-coordinate of a point of $E_{1}(\mathbb{Q})$. The roots of this polynomial are the $x$-coordinates of points of infinite order of $E_{t}(\mathbb{Q})$.

\section{References}

[A-M] A. O. L. Atkin and F. Morain, Finding suitable curves for the elliptic curve method of factorization, Math. Comp. 60 (1993), 399-405.

[Cas] J. W. S. Cassels, Lectures on Elliptic Curves, London Math. Soc. Student Texts 24, 1991.

[Fer] S. Fermigier, Exemples de courbes elliptiques de grand rang sur $\mathbb{Q}(t)$ et sur $\mathbb{Q}$ possédant des points d'ordre 2, C. R. Acad. Sci. Paris Sér. I 322 (1996), 949-952.

[Kna] A. Knapp, Elliptic Curves, Math. Notes, Princeton Univ. Press, 1992.

[Kub] D. S. Kubert, Universal bounds on the torsion of elliptic curves, Proc. London Math. Soc. (3) 33 (1976), 193-237. 
[Len] H. W. Lenstra, Jr., Factoring integers with elliptic curves, Ann. of Math. (2) 126 (1987), 649-673.

[Maz] B. Mazur, Rational isogenies of prime degree, Invent. Math. 44 (1978), 129-169.

[Mes1] J.-F. Mestre, Courbes elliptiques de rang $\geq 11$ sur $\mathbb{Q}(t)$, C. R. Acad. Sci. Paris Sér. I 313 (1991), 139-142.

[Mes2] -, Courbes elliptiques de rang $\geq 12$ sur $\mathbb{Q}(t)$, ibid. 313 (1991), 171-174.

[Mon] P. L. Montgomery, Speeding the Pollard and elliptic curve $m$ and hods of factorization, Math. Comp. 48 (1987), 243-264.

[Nag] K. Nagao, Construction of high-rank elliptic curves with a non-trivial torsion point, ibid. 66 (1997), 411-415.

[Na] T. Nagell, Recherches sur l'arithmétique des cubiques planes du premier genre dans un domaine de rationalité quelconque, Nova Acta Soc. Sci. Upsal. (4) 15 (1952), no. 6 .

[Sil] J. H. Silverman, Advanced Topics in the Arithmetic of Elliptic Curves, Grad. Texts in Math. 151, Springer, New York, 1994.

[Suy] H. Suyama, informal preliminary report, 1985.

Universidad Nacional de General Sarmiento

Arenales 3675 9P

1425 Capital Federal, Argentina

E-mail: lkulesz@ungs.edu.ar

kulesz@math.jussieu.fr

Received on 20.7.2001

and in revised form on 7.5.2002

(4079) 